UCRL-ID-133226

\title{
Portable Implementation of Implicit Methods for the UEDGE and BOUT Codes on Parallel Computers
}

\author{
T.D. Rognlien \\ X.Q. Xu
}

February 17, 1999

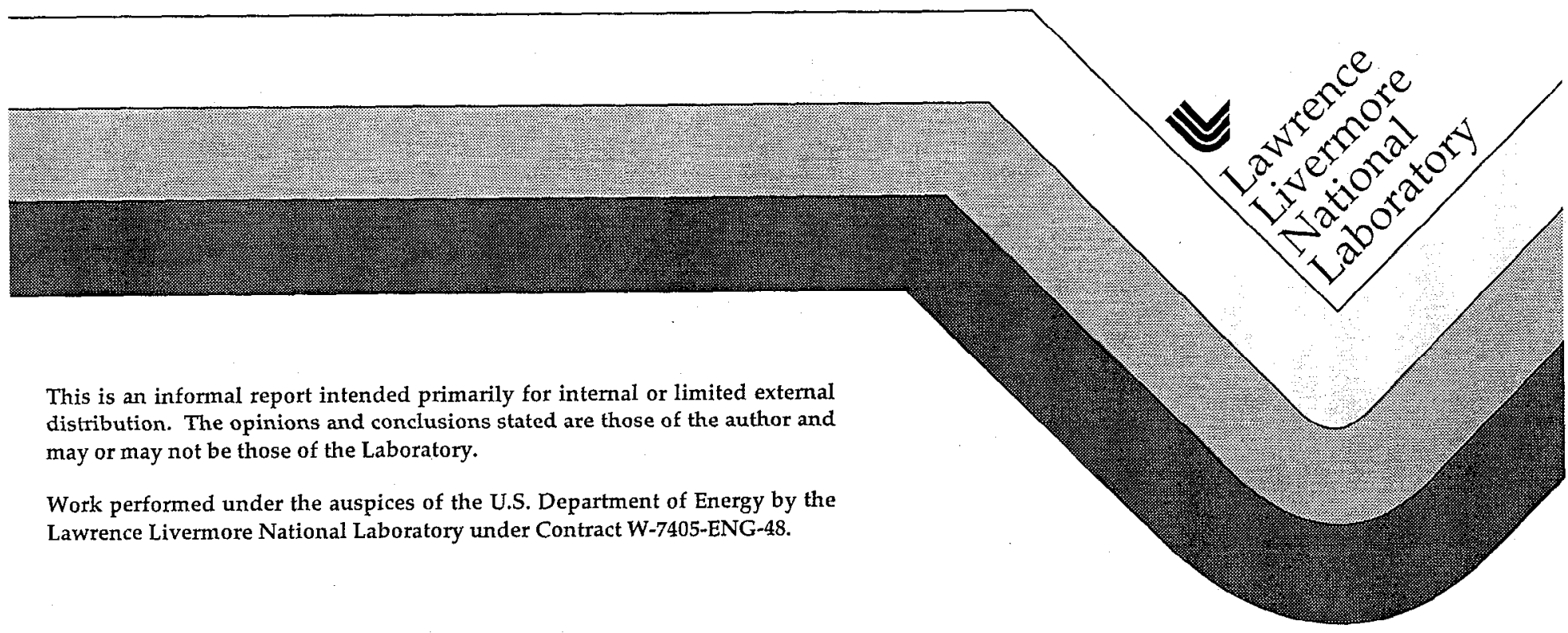




\section{DISCLAIMER}

This document was prepared as an account of work sponsored by an agency of the United States Government. Neither the United States Government nor the University of California nor any of their employees, makes any warranty, express or implied, or assumes any legal liability or responsibility for the accuracy, completeness, or usefulness of any information, apparatus, product, or process disclosed, or represents that its use would not infringe privately owned rights. Reference herein to any specific commercial product, process, or service by trade name, trademark, manufacturer, or otherwise, does not necessarily constitute or imply its endorsement, recommendation, or favoring by the United States Government or the University of California. The views and opinions of authors expressed herein do not necessarily state or reflect those of the United States Government or the University of California, and shall not be used for advertising or product endorsement purposes.

This report has been reproduced directly from the best available copy.

Available to DOE and DOE contractors from the Office of Scientific and Technical Information

P.O. Box 62, Oak Ridge, TN 37831

Prices available from (615) 576-8401, FTS 626-8401

Available to the public from the

National Technical Information Service

U.S. Department of Commerce

5285 Port Royal Rd.,

Springfield, VA 22161 
March 15, 1999

\title{
Portable Implementation of Implicit Methods for the UEDGE and BOUT Codes on Parallel Computers
}

\author{
T.D. Rognlien and X.Q. Xu \\ Lawrence Livermore National Laboratory \\ Livermore, CA 94551
}

A description is given of the parallelization algorithms and results for two codes used extensively to model edge-plasmas in magnetic fusion energy devices. The codes are UEDGE, which calculates two-dimensional plasma and neutral gas profiles, and BOUT, which calculates three-dimensional plasma turbulence using experimental or UEDGE profiles. Both codes describe the plasma behavior using fluid equations. A domain decomposition model is used for parallelization by dividing the global spatial simulation region into a set of domains. This approach allows the used of two recently developed LLNL Newton-Krylov numerical solvers, PVODE and KINSOL. Results show an order of magnitude speed up in execution time for the plasma equations with UEDGE. A problem which is identified for UEDGE is the solution of the fluid gas equations on a highly anisotropic mesh. The speed up of BOUT is closer to two orders of magnitude, especially if one includes the initial improvement from switching to the fully implicit Newton-Krylov solver. The turbulent transport coefficients obtained from BOUT guide the use of anomalous transport models within UEDGE, with the eventual goal of a self-consistent coupling. 


\section{Introduction}

The goal of this work is to develop the first numerical codes for simulation of edge plasmas for magnetic fusion energy (MFE) devices that exploit the power of parallel computers. Understanding edge plasmas is central to the problems of high heat flux from plasma power exhaust, adequate helium removal, and maintaining sufficient edge temperature, all recognized as critical issues for magnetic fusion reactors. Proper assessment of these issues requires detailed computer codes. The last several years has witnessed a period of unprecedented growth in the computer power available for modeling physical problems. To utilize this computational power, one needs to make the paradigm shift from coding for single-processors to coding for multi-processor computers. For codes with explicit time advancement, this shift is relatively straightforward because only local quantities enter expressions for the solution at each time step. However, many physical problems, including fusion edge plasmas, contain various phenomena that yield a wide range of time scales which render the equations describing them "stiff" in the numerical sense. Here, implicit methods are especially useful, but implicit methods are inherently nonlocal spatially and thus present a bigger challenge for parallelization compared to explicit methods.

The simulation of edge-plasmas has many time scales because of the simultaneous modeling of ion and electron transport along and across a confining magnetic field, together with neutral particle processes. Here we describe the parallelization of two codes that simulate the edge-plasma region: $\mathrm{UEDGE}^{1}$ solves for the two-dimensional (2-D) profiles of a multi-species plasma and neutrals given some anomalous cross-field diffusion coefficients, and $\mathrm{BOUT}^{2}$ solves for the three-dimensional (3-D) turbulence that gives rise to the anomalous diffusion. These two codes are thus complementary in solving different aspects of the edge-plasma transport problem; UEDGE needs BOUT's turbulent transport results, and BOUT nceds UEDGE's plasma profiles. Each code can take from a day to wecks on singleprocessor computers for large problems. An essential step to coupling these calculations is speeding up their individual execution times since many iterations may be needed for a consistent solution.

This parallelization work benefits greatly from the development of two parallel implicit solvers by Hindmarsh and Taylor: ${ }^{3}$ PVODE solves a system of time dependent ordinary 
differential equations, and KINSOL solves a system of nonlinear equations typically aimed at finding steady-state solutions. The serial versions of these Newton-Krylov solvers, VODPK ${ }^{4}$ and NKSOL,${ }^{5}$ have been used very productively for the serial version of UEDGE. However, for such solvers to work well for UEDGE, our experience on serial computers shows that the system of equations must be well preconditioned. The preconditioning step is to solve a closely related problem in a more efficient but approximate manner which, in effect, serves as a guess for the final solution. ${ }^{4,5}$ Thus, part of our work for UEDGE focuses on development of a parallel preconditioner based on a domain decomposition model. In addition, we have collaborated with Hindmarsh and Taylor ${ }^{3}$ to help debug and test interface routines that allow us to run UEDGE, written in FORTRAN, with PVODE and KINSOL, written in C, on a variety of parallel computer platforms from the massively parallel $\mathrm{T} 3 \mathrm{E}$ computer to shared-memory workstations with multiple processors (SUN and DEC). This aspect of our work demonstrates, for a complex problem, how one can reuse existing FORTRAN coding, and with moderate extensions, utilize recently developed implicit parallel solvers.

Another element of portability is provided by implementing message passing between multiple processors by using the MPI package. ${ }^{6}$ This package is available on many different platforms and allows one to utilize either shared memory or individual processor memory without changing the code. With MPI, one can now use processors on one computer or a network of computers. Also, the serial version of UEDGE has been made very productive through the use the BASIS system, ${ }^{9}$ but this programming and computing environment is not available on all computer platforms. This problem has been alleviated by recent development by Grote ${ }^{11}$ of a conversion capability from BASIS to the more widely available PYTHON system, ${ }^{10}$ and application of the conversion to UEDGE by Yang. ${ }^{12}$

The computational time required for the BOUT code exceeds that of UEDGE because BOUT simulates short to moderate wavelength turbulence in $3-\mathrm{D}$. We have made more than an order of magnitude gain in speed for BOUT by converting to the fully implicit solver PVODE even on a single processor (the single processor version is called CVODE). In contrast to UEDGE which seeks steady-state solutions, it is found that the large BOUT speed up is possible even without, a preconditioner, in part because one needs to maintain a time step to resolve the fluctuation time-scales of the turbulence. However, we believe BOUT could take even larger time steps with the aid of a proper preconditioner. To 
utilize parallel computers, we convert the serial implicit version of BOUT to a parallel domain-decomposition scheme similar to that of UEDGE which can use many processors simultaneously. The parallel version of BOUT also uses MPI for portability.

The plan of the reports is as follows: In Sec. II, the geometry and basic equation for the edge-plasma problem are given. The domain decomposition model used for UEDGE and BOUT is described in Sec. IIC. The results for the UEDGE parallelization are shown in Sec. III. The BOUT results from the fully implicit solver and parallelization are given in Sec. IV. The conclusions are summarized in Sec. V.

\section{Equations and Geometry}

\section{A. Basic equations}

The basic models in the UEDGE and BOUT codes are obtained from the plasma fluid equations of continuity, momentum, and thermal energy for both the electrons and ions as given by Braginskii. ${ }^{13}$ The continuity equations have the form

$$
\frac{\partial n}{\partial t}+\nabla \cdot\left(n_{e, i} \mathbf{v}_{e, i}\right)=S_{e, i}^{p}
$$

where $n_{e, i}$ and $\mathbf{v}_{e, i}$ are the electron and ion densities and mean velocities, respectively. The source term $S_{e, i}^{p}$ arises from ionization of neutral gas and recombination.

The momentum equations are given by

$$
n m_{e, i} \frac{\partial \mathbf{v}_{e, i}}{\partial t}+m_{e, i} n_{e, i} \mathbf{v}_{e, i} \cdot \nabla \mathbf{v}_{e, i}=-\nabla P_{e, i}+q n_{e, i}\left(\mathbf{E}+\mathbf{v}_{e, i} \times \mathbf{B} / c\right)-\mathbf{F}_{e, i}-\mathbf{R}_{e, i}+\mathbf{S}_{e, i}^{m}
$$

Here $m_{i}$ is the ion mass, $P_{e, i}=n_{e, i} T_{e, i}$ is the pressure with $T_{e, i}$ being the temperatures, $q$ is the particle charge, $\mathbf{E}$ is the electric field, $\mathbf{B}$ is the magnetic field, $c$ is the speed of light, $\mathbf{F}_{e, i}=\nabla \cdot \Pi_{e, i}$ is the viscous forcc, and $\mathbf{R}_{e, i}$ is the thermal force. ${ }^{13}$ The source $\mathbf{S}_{i, e}^{m}$ contains a sink term $-n m_{i, e} \mathrm{v}_{i} \dot{S}_{i, e}^{p}$ which arises if newly created particles have no drift motion.

The ion and electron energy equations can be written in the form

$$
\frac{3}{2} n \frac{\partial T_{e, i}}{\partial t}+\frac{3}{2} n \mathbf{v}_{e, i} \cdot \nabla T_{e, i}+P_{e, i} \nabla \cdot \mathbf{v}_{e, i}=-\nabla \cdot \mathbf{q}_{e, i}-\Pi_{e, i} \cdot \nabla \mathbf{v}_{e, i}+Q_{e, i}
$$

Here, $\mathbf{q}_{e, i}$ are the heat fluxes, and $Q_{e, i}$ are the volume heating terms. ${ }^{13}$ 
In their general three-dimensional form, the six equations given above represent ten separate partial differential equations for $n_{e}, n_{i}, \mathbf{v}_{e}, \mathbf{v}_{i}, T_{e}$, and $T_{i}$. UEDGE and BOUT use somewhat different assumptions to reduce the complexity of their models. The largest difference as discussed in Sec. B is that UEDGE assumes symmetry in a third dimension, usually the toroidal direction for a fusion device, whereas BOUT allows fluctuations to have variations in all 3 spatial dimensions even though the equilibrium profiles are toroidally symmetric.

\section{B. Geometry}

Both UEDGE and BOUT are written in general coordinates that can be adopted to a slab, cylinder, or torus by the use of the appropriate metric coefficients. For MFE fusion devices, we have been most interested recently in toroidal devices with an emphasis on tokamaks. The region occupied by the edge plasma for the poloidal plane of a tokamak with a single-null divertor is shown in Fig. 1. The long, sometimes closed lines of the mesh represent poloidal magnetic flux surfaces in which the magnetic field vector lies. For tokamaks, the strongest magnetic field component is in the toroidal direction, out of the plane of the figure.

UEDGE and BOUT use the the poloidal flux surfaces as one spatial coordinate. The second spatial coordinate is often the curves normal to the flux surfaces also shown in Fig. 1a, but a nonorthogonal mesh is sometimes used to form the mesh along material surfaces at the boundary. For UEDGE, this specifies the 2-D coordinate system and all quantities are assumed uniform in the third direction. However, BOUT allows fluctuations to arise in the third toroidal direction, even though the equilibrium is uniform. Here, a segment of the torus is simulated as shown in Fig. 1b which is assumed to be periodically replicated to fill out the full torus. Thus, the wavelength of the longest toroidal mode is set by the length of the toroidal segment used.

The numerical discretization schemes used by UEDGE and BOUT are similar in the two dimensions in the poloidal plane. UEDGE uses a conservative finite-volume method and BOUT uses a finite difference method including a fourth-order spatial discretization for the nonlinear $\mathrm{E} \times \mathrm{B}$ convective velocity terms. 


\section{Domain Decomposition Model}

Because UEDGE and BOUT use the same poloidal mesh, this region can be divided into domains on parallel computers where separate processors can solve a local problem. However, for the toroidal edge-plasma problem, there are a set of natural interior boundaries that need to be identified and accommodated for efficient decomposition. The regions delineated by these interior boundaries are shown in Fig. 2 for both the poloidal geometry and the corresponding mapping to a logically-rectangular domain. Information needs to be passed between cells that touch one of the dotted line between the private-flux and the core regions to the cells along the other dotted line, and vice versa. These interior boundaries are used to account for the periodic boundary conditions used within the core region and the continuity-of-flux condition from the private-flux region 3 to private-flux region 4 .

If the selection of the domains is such that the boundaries of major regions 1-4 in Fig. 2 always comprised of boundaries of the domains, then the finite difference representation in a given domain can be entirely local. Such a domain decomposition is shown in Fig. 3a where sixteen domains are used for the poloidal plane.

The information needed to form the local finite-difference approximations to the deriva-

tives at the boundary of the domains is provided by passing the variable data between processors (domains) via MPI ${ }^{6}$ in order to fill the guard cells that surround each domain shown in Fig. 3b. Notice that data needed in a guard cell is nol necessarily from the adjacent domain; e.g., the guard cell data for domain 0 in Fig. 3a comes from domain 3. These guard cells do not contain variables that are advanced for each domain, but rather contain a copy of this information from another processors. The only exception to this rule is for UEDGE which uses exterior guard cells to specify boundary conditions; but here the boundaries conditions are local, so no message-passing between domains is required.

The domain decomposition plays two roles. First, in order to utilize the implicit PVODE and KINSOL solvers, ${ }^{3}$ we must divide the physical space of our codes in this manner, where each processor has all of the information required to solve the local problem. Here we collaborated with Hindmarsh and Taylor to help debug and test interfaces (often called wrappers) between their solvers written in $\mathrm{C}$ and FORTRAN application programs like UEDGE that will run on a variety of parallel platforms. Because these Newton-Krylov 
implicit solvers use a local, finite-difference approximation to the Jacobian, there is no need to invert a global matrix. The second use of the domain decomposition model is that it provides the basis for the preconditioning algorithm which is required by UEDGE. Here the full set of Jacobian elements can be efficiently generated in parallel using all of the processors. However, to obtain a preconditioner, one needs to approximate the inversion of the Jacobian which is more nonlocal than the Krylov approximation. To do this, we perform an approximate LU decomposition of the Jacobian using ILUT ${ }^{7}$ routines independently on each processor. This procedure of not including coupling between domains at the preconditioning level is often referred to as the additive-Schwartz method. ${ }^{8}$

The manner in which UEDGE utilizes the solvers PVODE and KINSOL can be most succinctly explained by the diagram in Fig. 4 . On the left is the main UEDGE calculation of the finite-difference equations that yield the "right-hand side" of the evolutionary equations for each variable. In addition, UEDGE provides a local preconditioning Jacobian on each processor by approximating derivatives with finite-difference quotients. This operation should not be confused with the finite-difference approximation used by the PVODE and KINSOL Krylov solvers. In the central column of the diagram is the wrappers mentioned in the previous paragraph which pass data from the Fortran UEDGE code to the $\mathrm{C}$ solvers, and vice versa. Finally, on the right is the C solvers, PVODE or KINSOL, themselves. This section required no development on our part, and can thus be considered as "off-the-self" software. Note that the foregoing model is replicated for all domains or processors. Communication between processors as required to fill that guard cells is shown by the "mpi send and receive" boxes in Fig. 4.

The parallel model for BOUT is very similar to that just described for UEDGE, except that BOUT is written in $\mathrm{C}$ and thus requires no extra interface routines to utilize the $\mathrm{C}$ solvers. Since BOUT must follow the time-dependence, only the PVODE option is used here. Also, as mentioned earlier, BOUT works surprisingly well without a preconditioner. Some work has been done on testing preconditioners for even more improvement, but more development is needed here. 


\section{Implementation and Results for UEDGE}

\section{A. Implementation}

The 2-D plasma transport equations used in UEDGE come from a reduction of those presented in Sec. IIA for the parameters of the edge plasma. This results in five equations of the variables of ion density, $n_{i}$, ion parallel velocity, $v_{\|}$, separate electron and ion temperatures, $T_{e}$ and $T_{i}$, and the electrostatic potential, $\phi$. In addition, impurity species can be included which have their own density and parallel velocities. Furthermore, neutral gas species are present which can be described by various models; the simplest is a diffusion model, where the neutral density, $n_{n}$, obeys the continuity equation

$$
\frac{\partial}{\partial t} n_{n}+\frac{\partial}{\partial x}\left(n_{n} v_{n x}\right)+\frac{\partial}{\partial y}\left(n_{n} v_{n y}\right)=\left(\left\langle\sigma_{r} v_{e}\right\rangle-\left\langle\sigma_{i} v_{e}\right\rangle\right) n_{e} n_{n}
$$

Here the neutral velocities, $v_{n x, y}$, are taken from a diffusion approximation using the chargeexchange collision frequency between ions and neutrals, and the source and sink terms on the right-hand side represent recombination and ionization with rate coefficients $\left\langle\sigma_{r} v_{e}\right\rangle$ and $\left\langle\sigma_{i} v_{e}\right\rangle$, respectively. We find that this neutral gas equation plays a major role in the parallelization work for UEDGE, a point we will return to in the results section.

With UEDGF, we seek steady-state solutions in as efficient a manner as possible, while still retaining the options to simulate time dependent phenomena when needed. Using large time steps, or performing nonlinear iterations to steady state with no time step requires the use of a good preconditioner. The most effective preconditioner is forming the full Jacobian matrix by finite-difference quotients as mentioned previously. We now have two options for the parallel UEDGE, either using an algorithm developed within UEDGE, or because each domain with guard cells is now self-contained, we can use the band-blockdiagonal preconditioners PVBBDPRE and KINBBDPRE supplied as part of the PVODE and KINSOL routines, respectively. ${ }^{3}$

To implement the domain decomposition model, we have written routine that automatically divides the global mesh in a manner that respects the "natural" boundaries shown in Fig. 2. One can specify the number of sub-domains in each of these regions, and the algorithm works to optimize the load balancing by having any imbalance in the number of equations per domain being relegated to a minimum number of processors which do less 
work than the average. In this way, most processors do not need to wait for the few unbalanced ones to finish. Usually, we strive for complete load balancing by a proper choice of mesh sizes and number of domains. The newly written routine also sorts through the indexing for the guard cells and provides a map to specify which processors must communicate boundary data to each other. A set of routines were developed that deal with passing data from the master processor to domain processors. This data includes the initial guess to the global solution, the global geometrical data, and the mapping index for the guard cells needed for each domain. A similar routine is used to gather the data from all the processors into a global solution at the end of the run. Finally, another set of message-passing routines was constructed to refresh the guard cell date at the appropriate times during the Jacobian and Newton-Krylov steps.

\section{B. Results}

We have run UEDGE on the T3E-600 using the 16 domain configuration shown in Fig. 3 for the full DIII-D tokamak geometry in Fig. 1a. The execution time normalized to that for one processors is presented in Fig. 5. Here PVODE was used to run to steady state with the plasma equations and two different preconditioners where used, the case marked $X$ being PVBBDPRE, and the + point being the internal UEDGE preconditioner. In the table below the figure, the tabulated data shows the number of function (or right-hand side) evaluations, the number of preconditioners, the normalized time, and the ideal time. Although the speed of the calculation depends somewhat on the preconditioner used, experience with various approximate preconditioners on serial computers indicates that both are working well; errors in the preconditioner typically result in an inability to obtain a solution with UEDGE.

The difference in the speed up results from the two preconditioners in Fig. 5 is due primarily to the frequency with which they are updated. ${ }^{14}$ Note from the table in Fig. 5 that the PVBBDPRE case (labeled $X$ ) has only about $1 / 3$ of the preconditioner evaluations compared to the + data point with the UEDGE preconditioner. As a consequence, the $X$ data point has almost twice the number of overall function evaluations from PVODE. Thus, the results from the two preconditioners indicate the sensitivity of the trade-off between more frequent preconditioner evaluations (and LU decomposition), and fewer Newton-Krylov iterations as reflected in the function cvaluation count. The cxtra work required because the precon- 
ditioner is only solved locally on each domain and thus does not include domain-coupling information is reflected in the lower number of function and preconditioner evaluations for the 1 processor base-case.

While it is encouraging to obtain nearly an order of magnitude speed up for the plasma equations in UEDGE, the relatively simple gas equation shown by Eq. (4) proved more difficult. This problem has been traced back to the highly anisotropic mesh shown in Fig. $1 .^{15}$ This mesh is chosen to best represent the plasma which flows rapidly along the long flux surfaces and transports slowly across the magnetic flux surfaces owing to magnetic confinement. However, the gas evolving from the divertor plates does not experience a magnetic force and is not preferentially confined to the flux surfaces. We have studied this problem in some detail for a simple gas diffusion problem outside the actual tokamak geometry and find the same difficulty. We believe that providing more overlap information in the preconditioner should allow this problem to be overcome, such as using a Schur complement method. ${ }^{8,16}$ Also, when coupling to a Montc Carlo neutrals code for the gas description, ${ }^{17}$ this issue goes away, and one gets the added benefit that Monte Carlo codes parallelize very well. We are presently working on this parallel coupling.

\section{Implementation and Results for BOUT}

\section{A. Implementation}

For edge-plasma turbulence, the application of a fluid model is reasonable in part because of the low temperature and high collisionality. While the unstable modes can have wavelengths short compared to the scale lengths of equilibrium profiles, the dominant modes have perpendicular wavelengths which are larger than the ion gyroradius, $\rho_{s}$, consistent with a fluid approach. Thus, it is appropriate to use the Braginskii fluid equations as presented in Sec. IIA. By scaling arguments, the full set of fluid equations can be reduced to a sixvariable set for the electrostatic potential, $\phi$, magnetic vector potential, $A_{\|}$, plasma density, $n_{i}$, electron and ion temperatures, $T_{e}$ and $T_{i}$, and ion parallel velocity $v_{i \|}$. The parallel current, $j_{\|}$, and perpendicular vorticity, $\varpi$, are intermediate variables used to help solve the system. The equations for all of the variables time-evolutionary equations, except for 
$\phi$ and $A_{\|}$. These potentials satisfy similar equations:

$$
\begin{aligned}
\nabla_{\perp}^{2} \phi & =\varpi \\
\nabla_{\perp}^{2} A_{\|} & =-\frac{4 \pi}{c} j_{\|} .
\end{aligned}
$$

The $\phi$ potential equation is not obtained from Poisson's equation, but rather from the quasineutrality condition and the current continuity equation. Here $\nabla_{\perp}^{2}$ refers to the Laplacian operator in the directions perpendicular to the magnetic field. The solution to this rather simple looking equation that has important consequences for the parallel version of BOUT.

In order to efficiently simulate turbulence with short perpendicular wavelengths compared to parallel wavelengths (i.e., for wavenumbers $k_{\|} \ll k_{\perp}$ ), we choose field-line-aligned ballooning coordinates, $x, y$ and $z$, which are related to the usual flux coordinates $\psi$, $\theta$, and $\varphi$ by the relation $x=\psi-\psi_{s}, y=\theta, z=\varphi-\int q(x, y) d y$. The partial derivatives are: $d / d \psi=\partial / \partial x-\left(\int \partial q / \partial \psi\right) \partial / \partial z, d / d \theta=\partial / \partial y-q \partial / \partial z, d / d \varphi=\partial / \partial z$, and $\nabla_{\|}=\left(B_{p} / h B\right) \partial / \partial y$. The magnetic separatrix is denoted by $\psi=\psi_{s}$. Here the key ballooning assumption is $|\partial / \partial y| \ll|q \partial / \partial z|$ and $d / d \theta \simeq-q \partial / \partial z$. In this choice of coordinates, $y$, the poloidal angle, is also the coordinate along the field line.

In the most general case, the solution to Eqs. (5-6) requires a three-dimensional solver since one of the perpendicular directions is a composite of the poloidal and radial directions. However, utilization of the ballooning assumption with short toroidal wavelengths reduces the potential equations to two dimensions in the radial and toroidal directions. Since the potential equations then do not depend on the poloidal coordinate, it is efficient to divide the parallelization domain in this direction. The technique for solving Eqs. (5-6) is to FastFourier Transform (FFT) in toroidal direction and finite difference in the radial direction. Because these potential equations are linear, the solution for $\phi$ and $A_{\|}$only requires a tridiagonal inversion in the radial direction and the FFT; both operations are localized to each poloidal domain.

To study realistic problems, BOUT obtains magnetic geometry data and plasma profiles from global data files written by UEDGE. The magnetic data comes ultimately from an MHD equilibrium code and the plasma background profiles can be from a UEDGE solution or an analytic fit to experimental data. On a parallel machine, a pointer is set so that each 
processor only reads a subset of the data needed for its domain. Similarly, each processor writes and reads its own dumpfile for the data in its domain which can be used to restart or continue the problem. Presently, a restarted problem needs to use the same number of processors as the original problem. For post-processing, another program collects the data from a set of the dumped data files generated by BOUT, and generates a single file for the global solution.

\section{B. Results}

The first step to increase the speed of the BOUT code was to convert its ordinary differential equation (ODE) solver which advances the spatially discretized equations in time. Originally, an Adams predictor-corrector scheme was used. We then changed the time advancing algorithm to the Newton-Krylov method by using CVODE and the parallel PVODE. These new solvers also have an option for the Adams functional iteration method which is similar, but more implicit than the original predictor-corrector scheme. The comparison between the Newton-Krylov and Adams functional iterations on the allowable time step is dramatic as shown in Fig. 6 which gives the size of the time step as the simulation evolves in time from its initial conditions. At the beginning, both methods show similar time steps, but soon, the Krylov method is able to expand its time step by a factor of 50 compared to the Adams method for the same accuracy. In fact, this simulation includes the shear in the magnetic equilibrium near the X-point which was a problem that we could not integrate successfully with the previous predictor-corrector method. Thus, using the Newton-Krylov method has become an essential part of our generalized BOUT simulations. We have also found the same type of improvement of the Newton-Krylov method over the Adams functional iteration method for a simple 2-D, 2-species reaction-diffusion problem.

In order to extent these improvements to parallel machines, we developed a parallel version of BOUT based on domain decomposition as described in Sec. IIC. Because the potential equations, Eq. (5-6), are independent of the poloidal dimension in the ballooningcoordinate representation, the most effective choice of domains are those which segment the poloidal direction. Thus, in referring to Fig. 3, this would consist of removing the horizontal dotted lines, and combining domains $(0,4,8,12),(1,5,9,13)$, etc. Using these poloidal domains, the solution of Eqs. (5-6) can be done entirely on each domain without 
regard to the other domains. Then, only message-passing is required to fill the guard cells of each domain in order to use PVODE.

The effectiveness of the parallel Bout on a SUN Wildfire system is shown in Fig. 7. This parallel system has 16 processors per machine, 3 machines, and shared memory. Here and elsewhere, the speed up time refers to wall-clock time. Note that the speed up is nearly linear on one machine, with a small degradation at 15 processors ( 15 because of load balancing for this given problem). However, when going to 30 processors, the speed up drops dramatically. This is caused by either slowing message passing between machines or non-optimization of scheduling, issues which are being investigated. Also, we have used MPICH routines, but the use of SUN MPI may give improved performance on this SUN system. Nevertheless, the speed up with 15 processors is a factor of 13 , which is encouraging. Note that a point is also shown for the DEC cluster at LLNL for 1 processor. BOUT is somewhat faster than the SUN cluster for our problem using 1 processor, but more significantly, it is quite slow in the parallel mode because of scheduling issues which prevent us from obtaining even 10 processor on a given machine for a sustained period of time.

When this same problem is run on the T3E-900 at NERSC, one can more effectively study the behavior from 15 to 60 or more processors, and the results, shown in Fig. 8, are even more impressive. One can see that the speed up is actually super-linear over the range considered when normalized to the case using 5 processors which is the smallest number of processors we could fit this problem into. The super-linear behavior, or ofl-set linear at high processor number, is most likely caused by the different levels of CPU memory available on the T3E. For the $5 \mathrm{PE}$ case, the memory required per processor is significantly larger than that available in the fast cache memory, while for the 60 processor case, a larger percentage of the calculation can reside in the fast cache memory. The division of work for the 60 processor case is $81 \%$ for evaluating the BOUT physics equations, $12 \%$ for internal PVODE calculations, $6 \%$ for interprocessor MPI communications, and $1 \%$ for other overhead costs. The load balance between processors is very good with only a $\sim 1 \%$ variation. 


\section{Conclusions}

We have succeeded to develop parallel versions of two workhorse codes to simulate edge plasmas in MFE deviccs: UEDGE for 2-D transport and profilc cvolution, and BOUT for 3-D turbulence. Both codes solve the magnetized plasma fluid equations, with UEDGE focusing on long-time development of the plasma profiles and BOUT dealing with shorttime turbulence which causes anomalous radial transport. A similar domain decomposition model is used to achieve the parallelization where we then utilize the recently developed LLNL Newton-Krylov solvers PVODE and KINSOL. ${ }^{3}$

The parallelization of UEDGE has allowed us to obtain nearly an order of magnitude speed up in execution time for the plasma equations on 16 processors. ${ }^{15}$ Here we were able to reuse almost all of the original FORTRAN coding, although we did have to creatc a $B \Lambda$ SISfree version of the code that could run on the T3E; with the automated conversion ${ }^{11,12}$ to PYTHON, this should not be needed in the future. We developed a domain decomposition model including an automatic decomposition routine and a number of message passing routines, plus tested and debugged interface routines with the PVODE and KINSOL solvers. The fluid gas equations do not parallelize as effectively as the plasma equations which wo have identified as caused by the anisotropic mesh and lack of domain overlap in the preconditioner. There are overlap methods which should be assessed for this problem. Also, the coupling of the parallel plasma equations with a parallel neutral Monte Carlo code looks promising.

The results for the BOUT 3-D code have exceeded our initial expectations. The conversion to the Newton-Krylov solver ${ }^{3}$ has produced a code which runs as much as 50 times faster compared to a Adams functional iteration method. In fact, the previous predictor-corrector method we used, which is even simpler than the Adams functional iteration method, and could not be practically used for the simulations which include X-point shear from the equilibrium magnetic field. These simulations are very important for understanding the behavior of present experiments and designing future devices. ${ }^{18,19}$

The parallelized version of BOUT continues to work well with a poloidal domain decomposition, giving a factor of 13 speed up for 15 processors on the SUN Wildfire and a very encouraging factor of 69 speed up for 60 processors on the T3E-900. The degradation on the 
Wildfire system at 15 processors may be do to inefficient message passing by using MPICH and not SUN MPI; this is presently be checked. The super-linear speed up on the T3E is likely due to the better utilization of cache memory for the larger number of processors. Most recently, we have extended this case to 120 processor on the T3E, and find the data on the same off-set linear curve. The large specd up of BOUT gives real optimism concerning coupling UEDGE and BOUT. Previously, BOUT was very time consuming, which made such coupling seem far off; now it is a real possibility.

There are two areas where more short-term improvements may be realized with BOUT performance. One is to extend the domain decomposition to the radial direction as in UEDGE. This will allow more domains as the number of allowable toroidal modes increases. Here we will deal with the coupling of the potential equations across the radial domains by a parallel tridiagonal solver ${ }^{20}$ or an Newton-Krylov solver using a preconditioner. The second area being focused on is to increasc the time stcp of the PVODE integration further by providing a preconditioner for the time dependent equations. This gain does have limitations in that we must still properly resolve the turbulence. Some simple preconditioners where tried without much improvement, but we know from our experience with UEDGE that preconditioners can be effective for the equations set we are using, and this warrants further investigation.

Acknowledgments: This work was supported by the IDR.D program at LLNL during Fiscal Years 1997-98 as project 97-ERD-045. We have benefited from many useful discussions with A.C. Hindmarsh, A.G. Taylor, and P.N. Brown. The simulations on the SUN Wildfire and DEC computers were performed at LLNL while the simulations on the T3E-600 and T3E-900 computers were performed at NERSC. We thank the Livermore Computing Center staff and the NERSC staff for their able assistance. Chris H.Q. Ding from NERSC has been especially helpful. 


\section{References}

${ }^{1}$ T.D. Rognlien, P.N. Brown, R.B. Campbell, et al., "2-D Fluid Transport Simulations of Gaseous/Radiative Divertor," Contrib. Plasma Phys. 34, 362 (1994).

${ }^{2}$ Xueqiao Xu and Ronald H. Cohen, "Scrape-Off Layer Turbulence Theory and Simulations," Contrib. Plasma Phys. 38, 158 (1998).

${ }^{3}$ A.C. Hindmarsh and A.G. Taylor, "PVODE and KINSOL: Parallel Software for Differential and Nonlinear Systems," Lawrence Livermore National Laboratory Report UCRLID-129739, Feb. 1998.

${ }^{4}$ P.N. Brown and A.C. Hindmarsh, "Reduced Storage Matrix Methods in Still ODE Systems," J. Appl. Math. Comp. 31, 40 (1989).

${ }^{5}$ P.N. Brown and Y. Saad, "Hybrid Krylov Methods for Nonlinear Systems of Equations," SIAM J. Sci. Stat. Comput. 11, 450 (1990).

${ }^{6}$ W.D. Gropp, E. Lusk, and A. Skjellum, Using MPI Portable Parallel Programming with the Message-Passing Interface, The MIT Press, Cambridge, MА, 1994.

${ }^{7}$ Y. Saad, "ILUT: A Dual Threshold Incomplete ILU Factorization," Num. Lin. Alg. Applic. 1, 387 (1994).

${ }^{8}$ Yossef Saad, Iterative Methods for Sparse Linear Systems, PWS Publishing Co., Boston, MA, 1996.

${ }^{9}$ P.F. Dubois, et al., "The Basis System", Lawrence Livermore National Laboratory Report UCRL-MA-118543, parts 1-6, 1994.

${ }^{10}$ Mark Lutz, Programming Python, O'Reilly \& Associates, Sebastopol, CA 1996.

${ }^{11}$ D.P. Grote, private communication, 1998.

${ }^{12}$ T-Y.B. Yang, private communication, 1998.

${ }^{13}$ S.I. Braginskii, "Transport Processes in a Plasma", in Revicws of Plasma Physics, Vol. I, Ed. M.A. Leontovich (Consultants Bureau, New York, 1965), p. 205. 
${ }^{14}$ T.D. Rognlien, X.Q. Xu, P.N. Brown, A.C. Hindmarsh, and A.G. Taylor, "Parallelization of an Edge Plasma Transport Code via Domain Decomposition," Bull. Am. Phys. Soc. 42, 1584 (1997).

${ }^{15}$ T.D. Rognlien, X.Q. Xu, A.C. Hindmarsh, P.N. Brown, and A.G. Taylor, "Algorithms and Results for a Parallelized Fully-Implicit Edge Plasma Code," Int. Conf. Num. Sim. Plasmas, Feb. 10-12, 1998, Santa Barbara, CA.; LLNL Report UCRL-JC-129223-abs.

${ }^{16}$ E.T. Chow, private communication, 1998.

${ }^{17}$ M.E. Rensink, L. LoDestro, G.D. Porter, T.D. Rognlien, and D.P. Coster, "A Comparison of Neutral Gas Models for Divertor Plasmas," Contrib. Plasma Phys. 38, 325 (1998).

${ }^{18}$ X.Q. Xu, R.H. Cohen, G.D. Porter, et al., "Turbulence in Boundary Plasmas," J. Nucl. Mater., to be published (1999).

${ }^{19}$ X.Q. X11, R.H. Cohen, G.D. Porter, et al., "Turbulence Studies in Tokamak Boundary Plasmas with Realistic Divertor Geometry," Proc. 17th Fusion Energy Conf., Yokohama, Japan, Oct. 19-24, 1998, paper IAEA-F1-CN-69/THP2/03; to be pub., IAEA (Vienna).

${ }^{20}$ N. Mattor, T.J. Williams, and D.W. Hewett, "Algorithm for Solving Tridiagonal Problems in Parallel," Parallel Computing 21, 1769 (1995). 


\section{Figures}

FIG. 1. The toroidal tokamak geometry simulated by the UEDGE and BOUT codes. In a), the poloidal plane plot shows the 2-D edge region simulated by UEDGE and the mesh used which has one coordinate based on magnetic flux surfaces as provided by an MHD equilibrium code. In addition to simulating the poloidal annulus in a), BOUT also allows fluctuations to have toroidal variations which fit periodically into the toroidal segment shown from the top view in b). Thus, inclusion on longer toroidal wavelength modes requires using a larger toroidal segment at increase computational cost.

FIG. 2. The poloidal plane is divided into 4 main regions for the domain decomposition model, each of which can be further subdivided. The 4 regions are mapped into the rectangular geometry shown in the lower part of the figure by opening the poloidal configuration along the dotted line.

FIG. 3. Division of UEDGE geometry into 16 regions is shown in a), while in b) more detail of mesh is shown within the domains and the overlapping guard cells.

FIG. 4. Schematic showing the three major components of the parallel UEDGE code as replicated on each domain or processor.

FIG. 5. Comparison of time to reach a steady state solution for the parallel UEDGE run on the T3E-600 parallel computer with 1 processor and 16 processors for the plasma equations with PVODE. The point labeled $X$ uses the PVBBDPRE preconditioner and the + point uses the internal UEDGE preconditioner. The table gives the number of function evaluations, preconditioner evaluations, and the normalized time to steady state.

FIG. 6. Time step allowed in BOUT over the course of a time dependent simulation showing the improvement obtained with new Krylov solver PVODE (or CVODE on serial computers) compared to the previously-used functional iteration method.

FIG. 7. Comparison of speed of parallel BOUT run on the LLNL SUN Wildfire system with 16 processors per machine. Only poloidal decomposition is used with no preconditioner. The drop from 15 to 30 processors is due to needed to run between two computers; may be due to slowing message passing or non-optimization of scheduling. 
FIG. 8. Comparison of speed of BOUT runs with various numbers of processors on the NERSC CRAY T3E-900. Only poloidal decomposition is used with no preconditioner. The super-linear behavior, or off-set linear curve, is likely caused by better utilization of fast cache memory at for a large number of processors. 


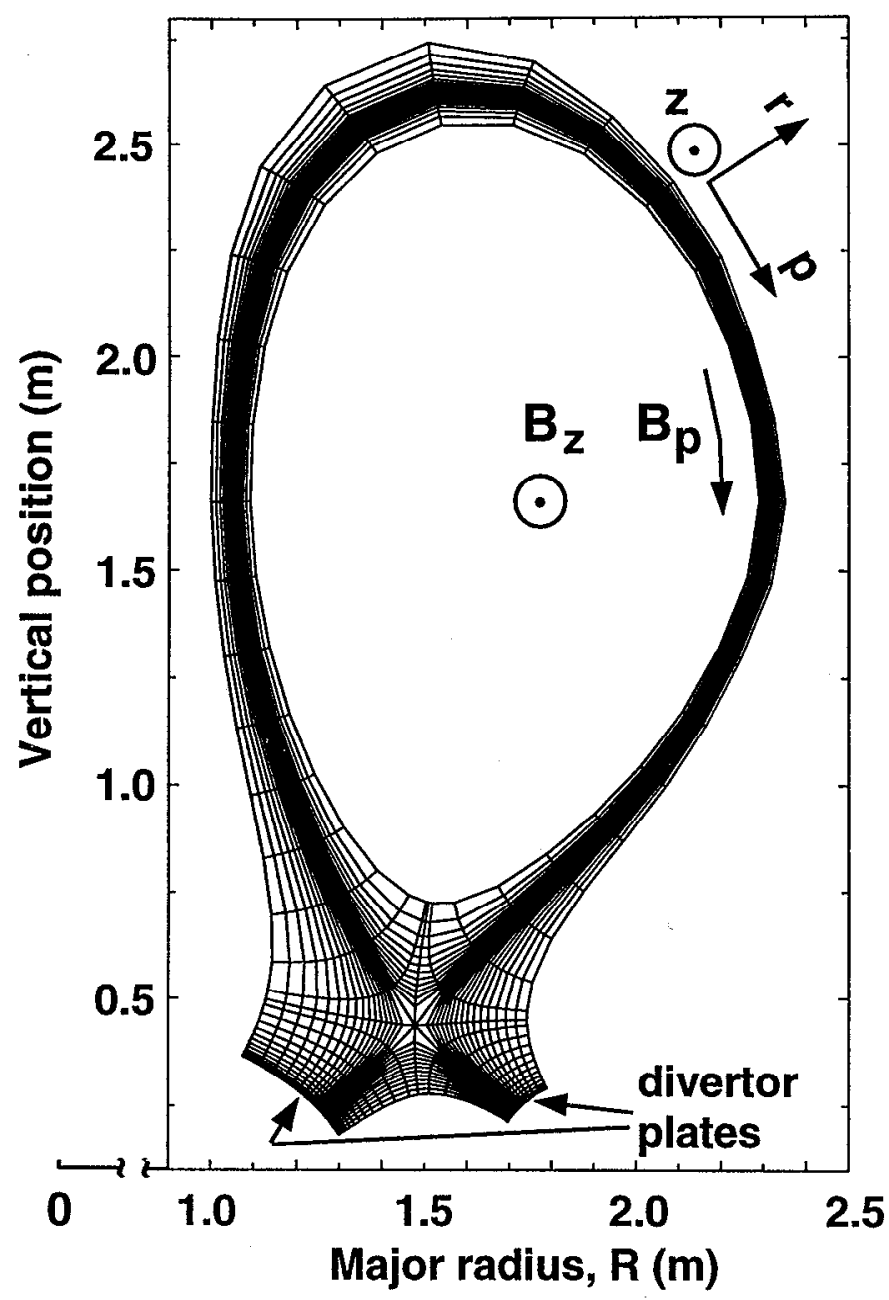

a)

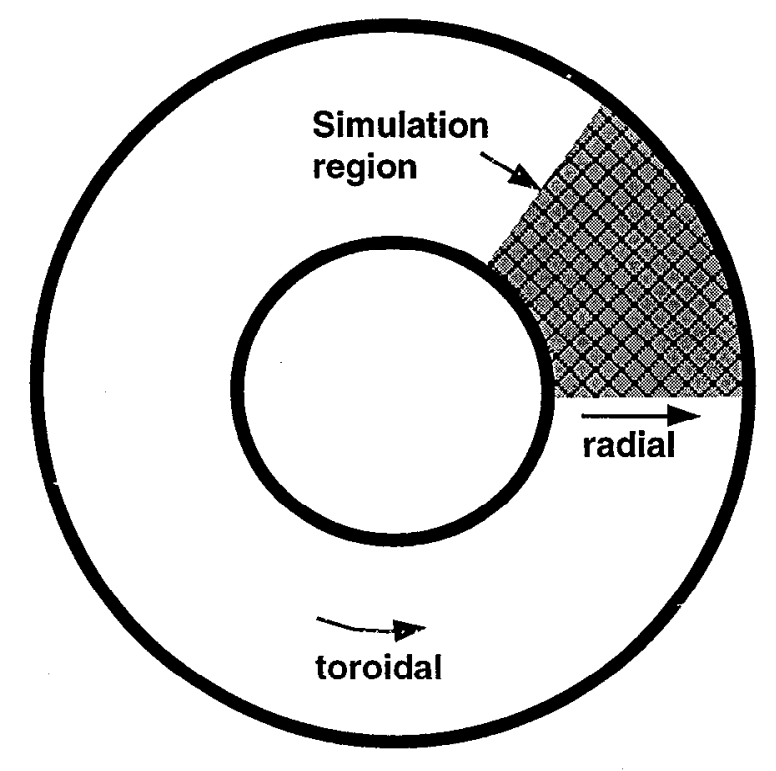

b)

Fig. 1 


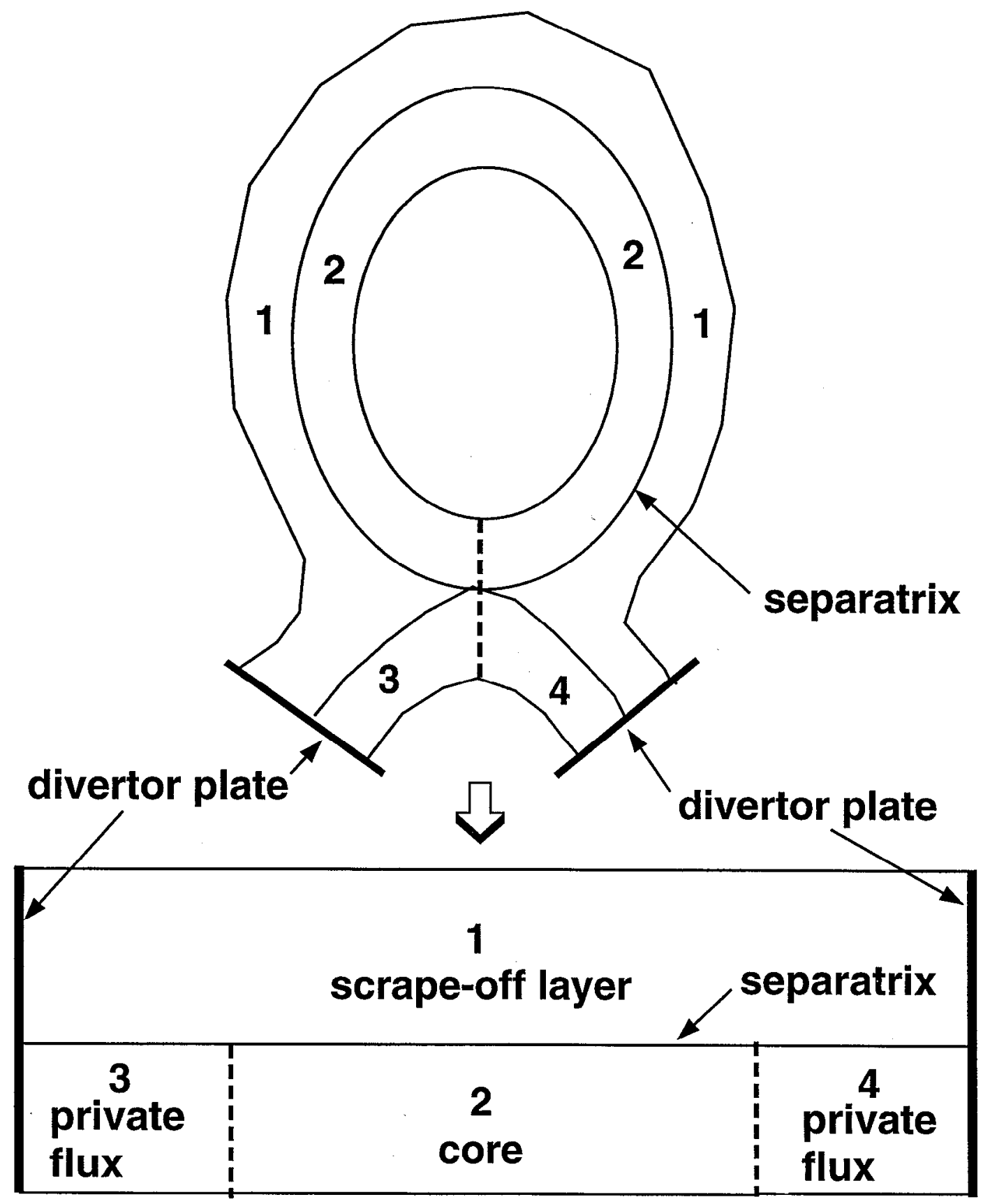

Fig. 2 
a)

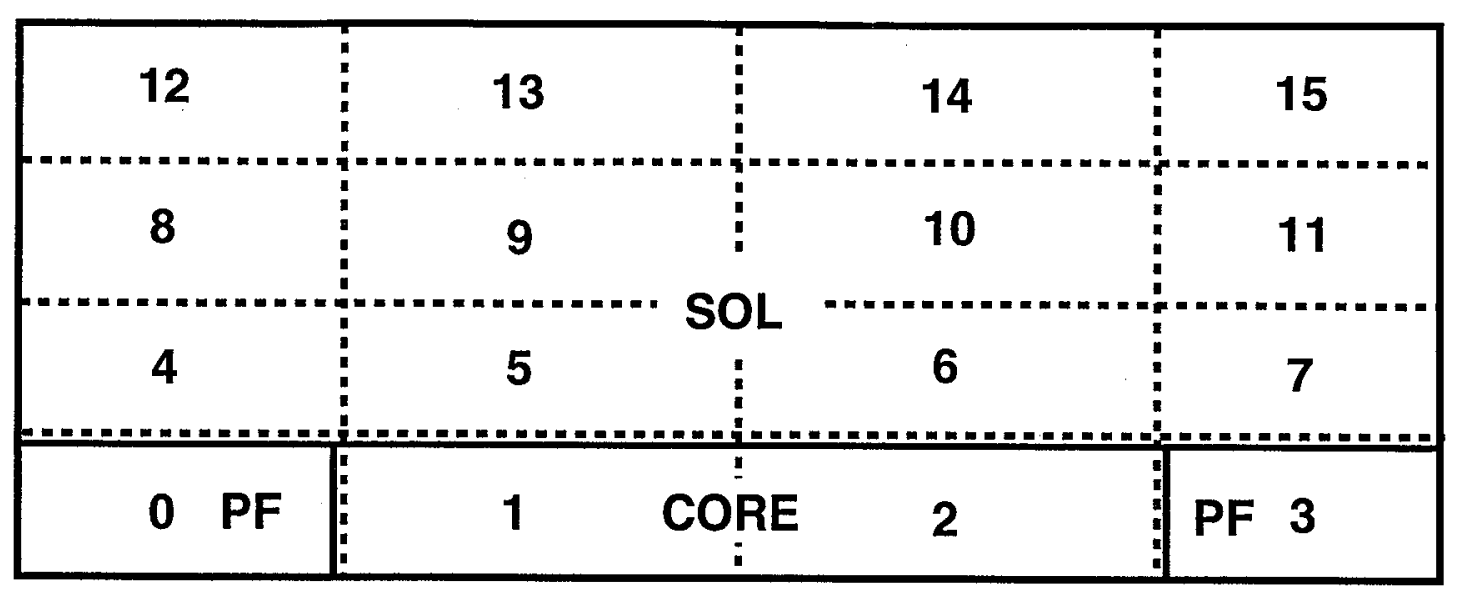

b)

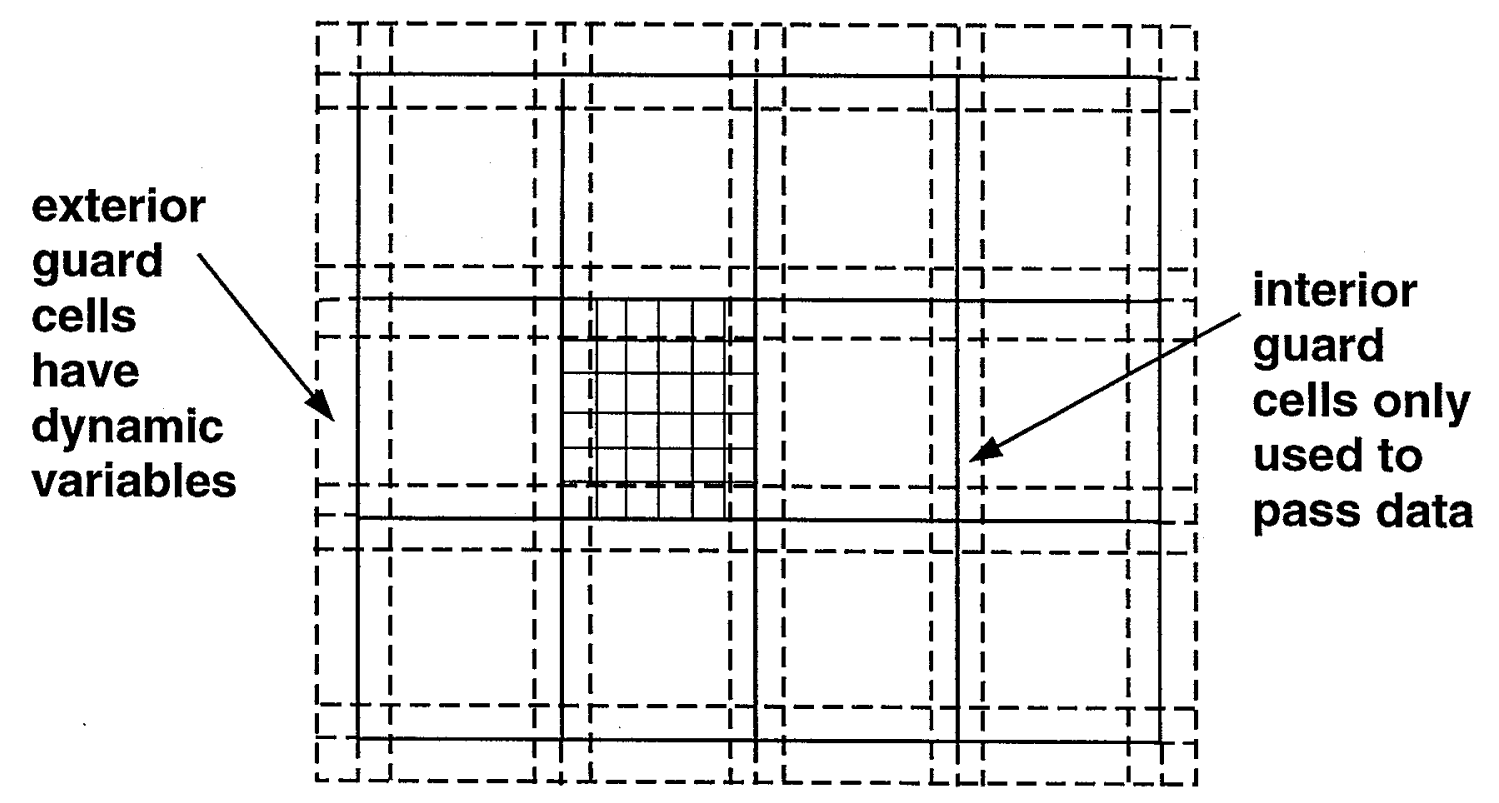

Fig. 3 
FORTRAN PHYSICS SOURCE

FORTRAN $<-->>C$

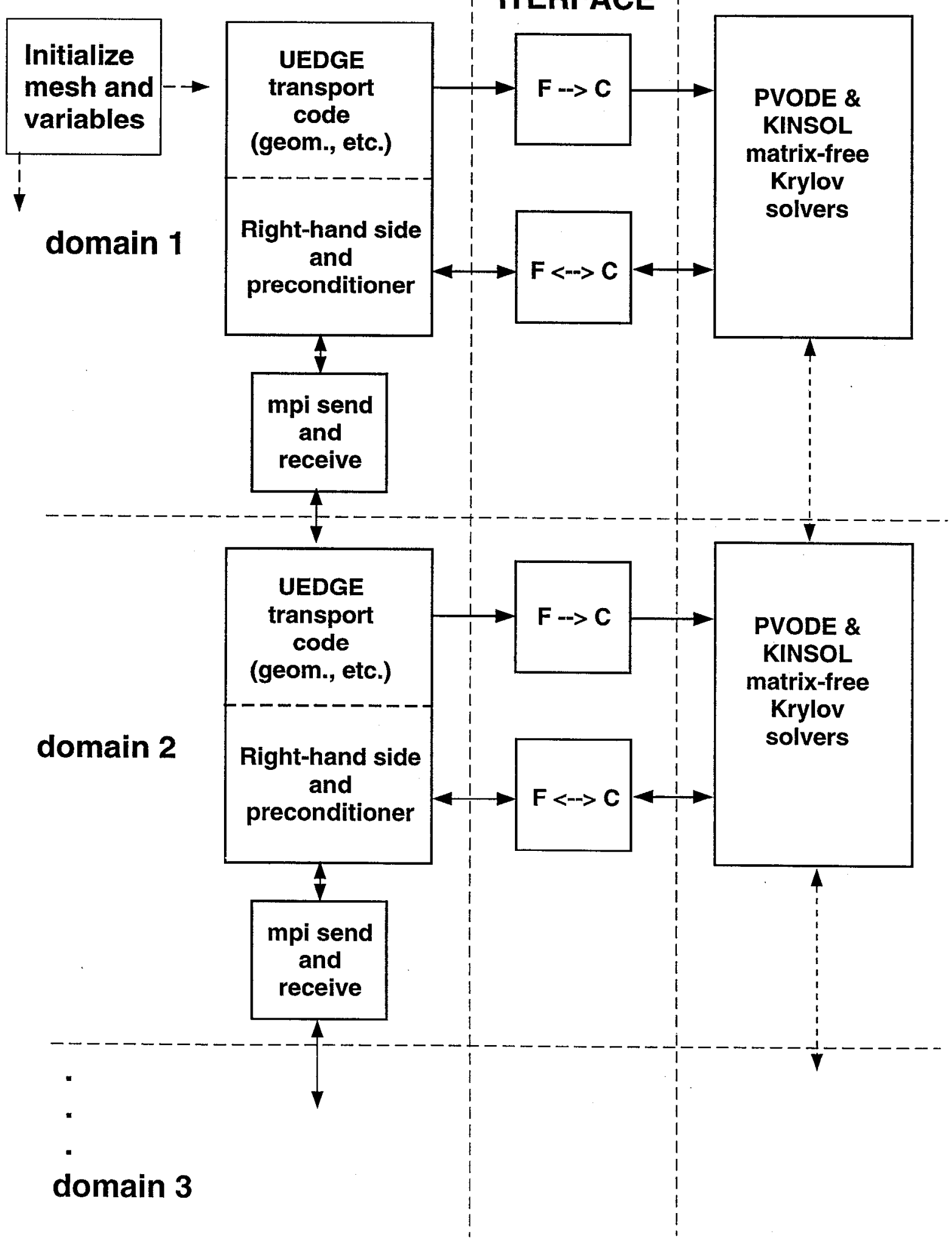

Fig. 4
C SOLVER

matrix-free

Krylov

solvers 


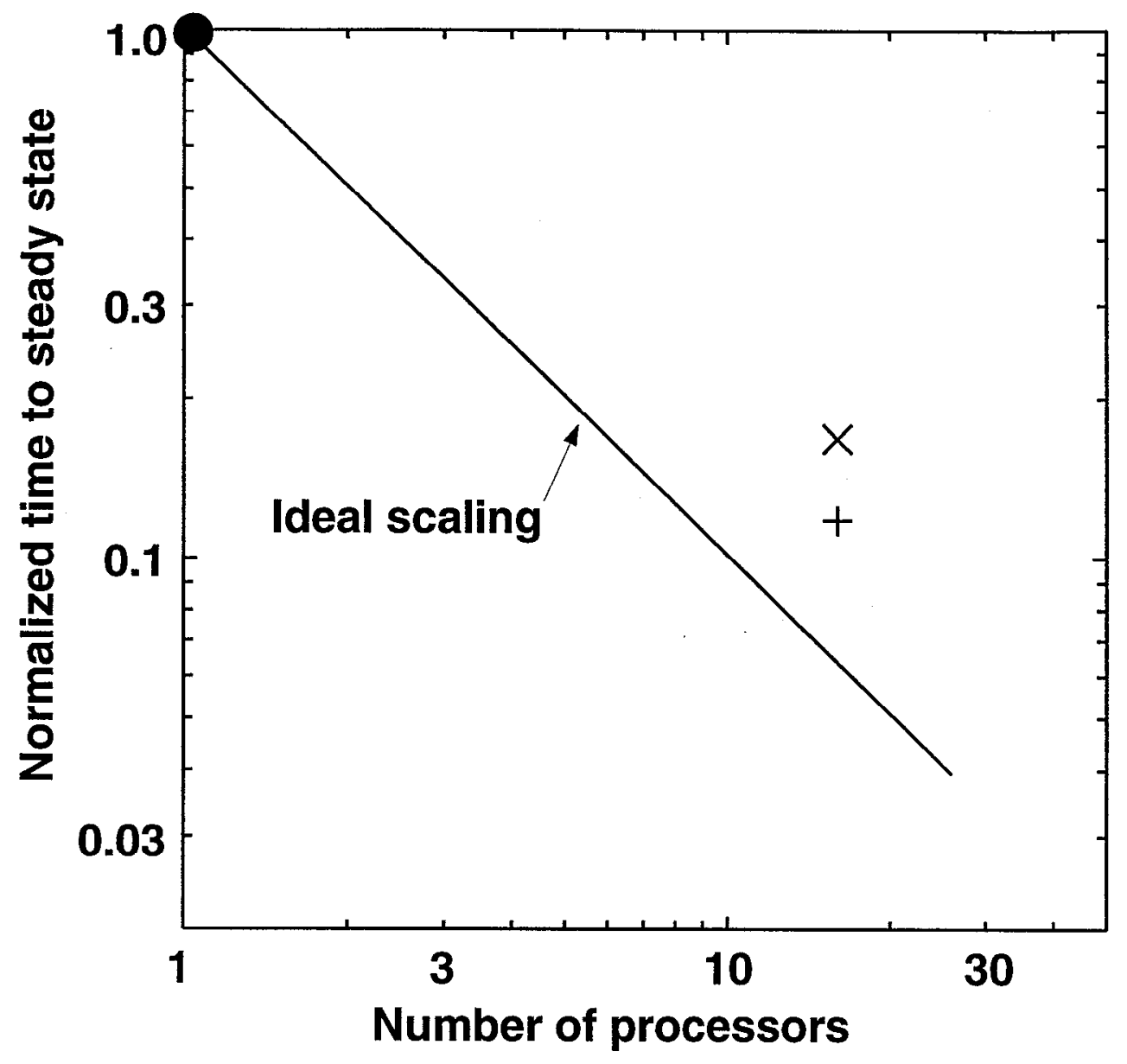

\begin{tabular}{|c|c|c|c|c|}
\hline Num. PEs & Func. eval & Prec. eval & Nor. time & Ideal time \\
\hline $1(O)$ & 2236 & 9 & 1.0 & 1.0 \\
\hline $16(\mathrm{x})$ & 9803 & 24 & 0.168 & 0.0625 \\
\hline $16(+)$ & 5760 & 67 & 0.118 & 0.0625 \\
\hline
\end{tabular}

Fig. 5 


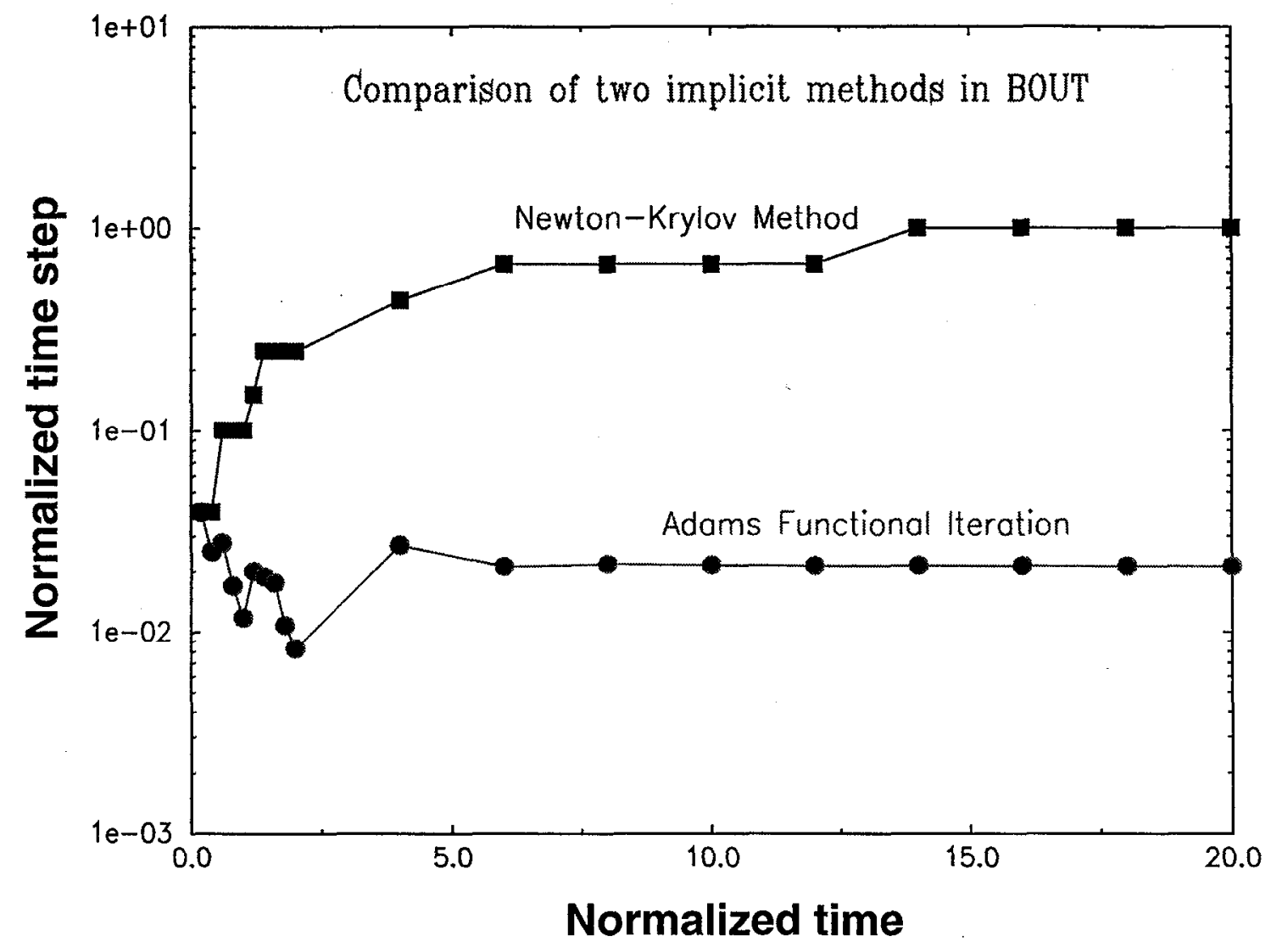

Fig. 6 


\section{Parallel Speed-up of BOUT on Sunbert}

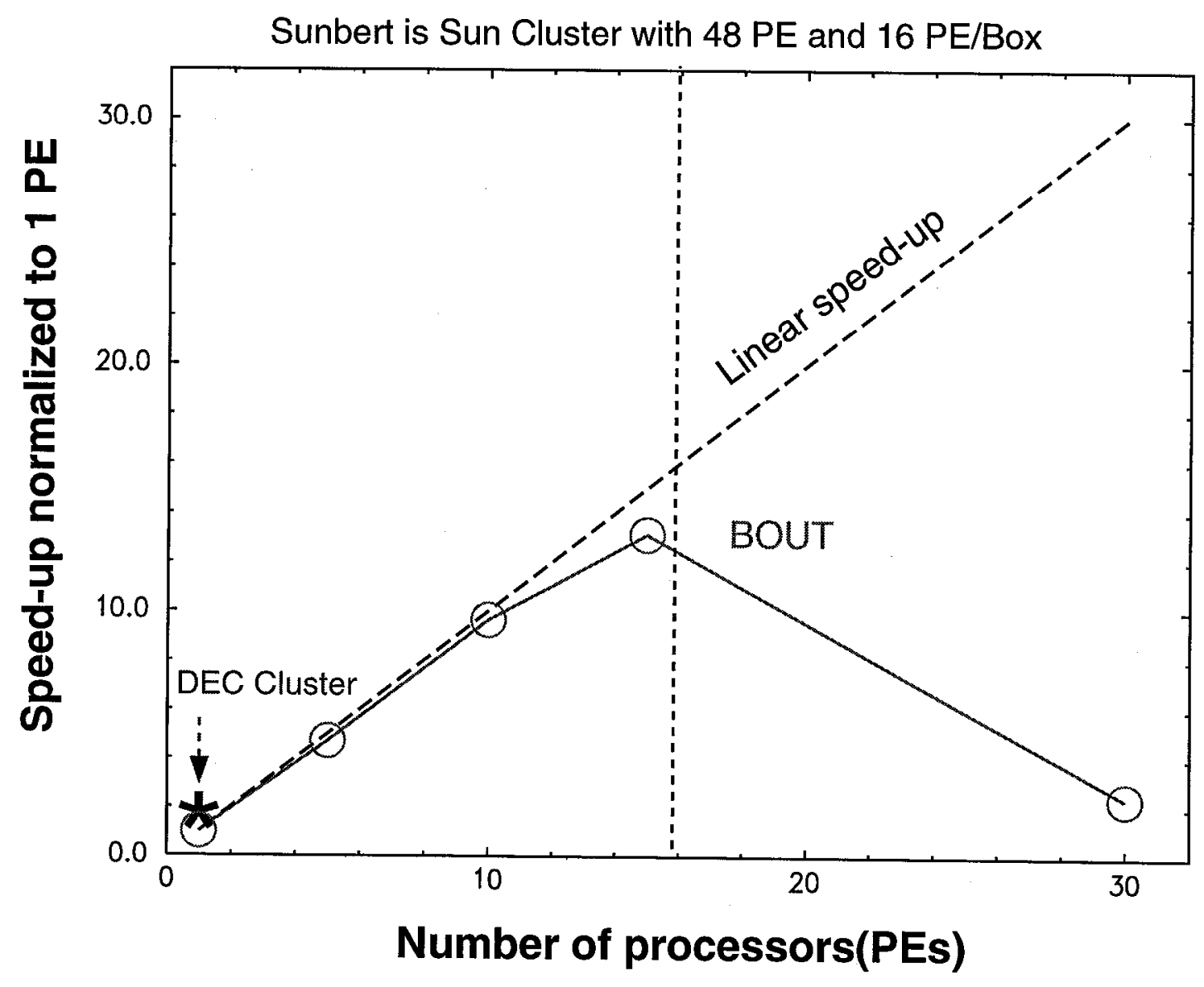

Fig. 7 


\section{Parallel Speed-up of BOUT on T3E}

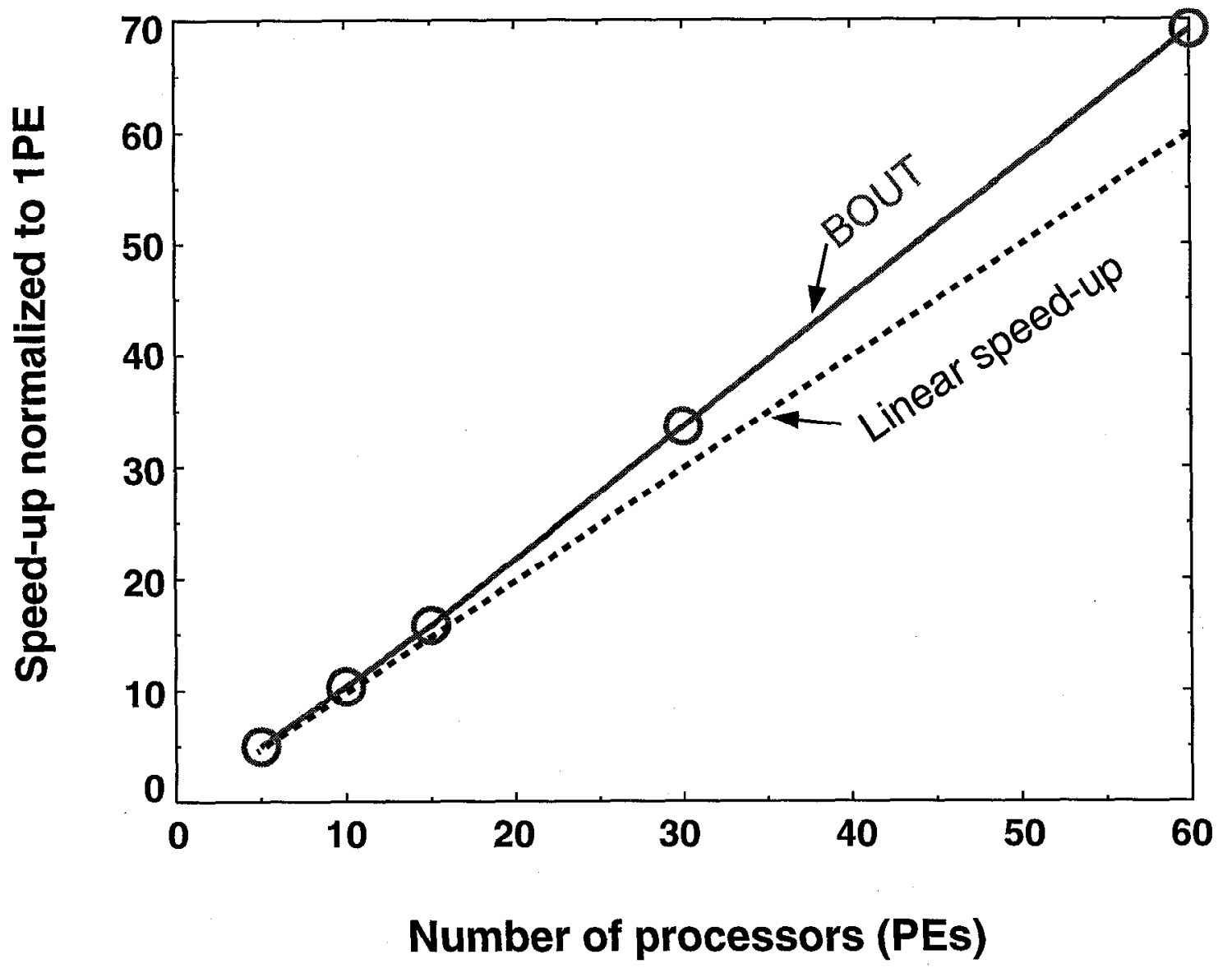

Fig. 8 